\title{
Optimizing assembly planning through a three-stage integrated approach
}

\author{
Ruey-Shun Chen ${ }^{\mathrm{a}}$, Kun-Yung Lu ${ }^{\mathrm{b}, *}$, Pei-Hao Tai ${ }^{\mathrm{c}}$ \\ a Institute of Information Management, National Chiao-Tung University, Taiwan \\ ${ }^{\mathrm{b}}$ National Lien-Ho Institute of Technology, 1 Lien Kung, Kung-Ching Li, Miao-Li, Taiwan \\ ${ }^{\mathrm{c}}$ Logistics Bureau, CSF, Taipei, Taiwan
}

Received 13 March 2003; accepted 30 May 2003

\begin{abstract}
This study proposes a three-stage integrated approach with heuristic working rules to assist the planner to develop a better assembly plan. In the first stage, Above Graph and transforming rules were used to create a correct Explosion Graph of the assembly models. In the second stage, a three-level relational model was developed to create a complete relational model graph and an incidence matrix. In the third stage, a mathematical model, based on a penalty index was formulated, and a revised minimum spanning table method was used to generate and evaluate a feasible assembly sequence. The example of a military system is presented to demonstrate the feasibility of the proposed method. The contribution of this study is based on a design for assembly potential applications of three-dimensional (3D) component models to assist manual or automatic assembly in a virtual environment, and allows the designer to recognize the relative position, constraints and relationships of 3D components using graph-oriented methods: Above Graph, assembly precedent diagram, and relational model graph.
\end{abstract}

(C) 2003 Elsevier B.V. All rights reserved.

Keywords: Assembly planning; Design for assembly; Minimum spanning tree; Assembly precedent diagram

\section{Introduction}

Assembly involves the integration of components and parts to produce a product or system. An effective assembly plan ensures the standard of RMA (reliability, maintainability and availability) during a product's lifecycle; this can increase production efficiency and reduce the cost of a product. Effective assembly planning facilitates

\footnotetext{
*Corresponding author. Fax: +886-3-7362-809.

E-mail addresses: rschen $@$ bis03.iim.nctu.edu.tw (R.-S. Chen), carlu@mail.nlhu.edu.tw (K.-Y. Lu), taiwy@, ml.is.net.tw (P.-H. Tai).
}

concurrent engineering in product development, operations and system analysis, to enhance the quality of a particular system. However, assembly planning is commonly a tedious design procedure requiring a significant amount of manpower to analyze the combination of relationships of each component or part (Tai, 1997). Rationalization of an assembly, including the development of new materials, time-and-motion studies, methods analysis and improvement, product development and design, etc., must be dedicated to assembly planning for improving the quality of the assembled product quality and for reducing its cost (Nof et al., 1997; Amen, 2001). 
In practice, a common approach to assembly planning is "assembly by disassembling", i.e. an assembly sequence results from systematically disassembling the final product and reversing the disassembling sequence (Lee, 1989). This recursive decomposition process may be represented by a non-ordered directed tree, which is called "assembly tree". It usually employs the contact-base feature to represent the precedence relationships of the product. A designer can successively assign the assembly relations to form the assembly plan based on the precedence diagram. However, the contact-base precedence diagram cannot effectively express the complexity of the assigned assembly relations. An effective assembly plan must include other graphs, such as the explosion graph, the relational model graph, the incidence matrix, the assembly precedent diagram (APD), etc. These graphs can be used to describe the assembly relations of the parts clearly. In reality, few experts or engineers know exactly how to derive a correct explosion graph, draw a complete relational model graph or incidence matrix among the components, or determine a complete APD diagram to generate an optimal assembly sequence. An assembly plan is normally implemented with the planner's common sense, past experience, and limited knowledge. Manual analysis does not allow the feasibility of assembly sequences to be easily verified. Furthermore, Kroll indicated that the planner frequently neglects the physical constraints of the assembly, which leads to difficulty in specifying an optimal assembly plan and which causes to a significant amount of time being spent in assembly planning (Kroll, 1994).

A good assembly plan must simultaneously consider crucial factors, such as physical and geometrical constraints, the similarity of assembly operations, the frequency of tool replacement, etc. This study presents a three-stage integrated approach with some heuristic working rules to assist the planner to obtain a better assembly plan. First, by employing 2D engineering drawings and related assembly information to create a correct Explosion Graph of the assembly models based on contact-above concepts and transforming rules; the Explosion Graph can be used to represent the correct geometric relationships among the assem- bly parts and simulate a 3D model of assembly. Second, a three-level relational model was developed to generate a complete relational model graph as well as the incidence matrix. The relational model graph can be advanced transformed into an APD diagram, which is used to describe the assembly sequences matching the precedent relations of the parts. Based on these graphs, the designer can easily find the feasible sequences and evaluate the difficulty of the assembly. Finally, an assembly difficulty rank method was employed to evaluate the penalty index of the respective assembly sequences, and then a revised minimum spanning table method (RMST) was used to minimize the total penalty in obtaining a feasible assembly plan.

The purpose of this paper is to generate an effective assembly plan to assist in modeling an assembly line and constructing the operation procedures in the maintenance fields. The main contribution of this paper is that the proposed approach allows the designer to simultaneously consider the crucial factors mentioned above to find an optimal solution through the evaluation of the penalty index of the assembly. This can facilitate the design for assembly (DFA) during the design phase.

The remainder of this paper is organized as follows. Section 2 discusses pertinent literature on assembly planning. Section 3 describes the proposed frameworks and transforming procedures. Section 4 describes the mathematical model and the algorithms of the RMST method. Section 5 then presents a case study to demonstrate the feasibility of the proposed approach. Discussion and conclusions are given in Section 6.

\section{Related work}

Recently, assembly line planning problems have received considerable attention in order to serve quickly and responsively service customers in a competitive environment. These studies can be classified as addressing one of a number of production optimization problems, including the wellknown assembly line balancing problem (ALB) (Ponnambalam et al., 2000; Kim et al., 1996; 
Amen, 2000), the tooling problem (Lazzerini and Marcelloni, 2000), and many routing and scheduling problems (Leu et al., 1996; Song et al., 2001). According to the research of Lucertini et al. (1998), effective assembly line modeling can reduce the number of tools or machines and can eliminate a bottleneck in a production line. Although many heuristic algorithms have been employed to solve these problems, a problem that involves $m$ workstations with $n$ tasks requires time $O\left(m^{2} n\right)$ to find the optimal solution (Lucertini et al., 1998). A typical NP-hard optimization problem with the complexity of precedence graphs cannot be solved problem in polynomial time (Ramos et al., 1998).

Assembly line modeling problems have been conventionally classified into two types-Type I and Type II (Hackman et al., 1989). In Type I problems, the required production rate (cycle time), assembly tasks, tasks times, and precedence requirements are given. Designing a new assembly line is generally such a problem, its objective being to minimize the number of workstations. In Type II problems, the number of workstations or production employees is fixed. The aim is to minimize the cycle time. Type II problems often occur when a factory wants to produce the optimum number of items using a fixed number of workstations without adding new machines. However, both types of assembly problems must rely on the given APD to formulate the assembly line.

Conventionally, optimal solutions of the ALB problems have typically been obtained by following a heuristic approach, such as those of maximum ranked positional weight (Helgeson and Birnie, 1961), maximum total number of follower tasks (Brian and Patterson, 1984), minimum total number of predecessor tasks (Elsayed and Boucher, 1994), maximum task time (Kilbridge and Wester, 1961), minimum reverse positional weight (Elsayed and Boucher, 1994), and others. Normally, these approaches can only solve the problem of a single model and deterministic task time.

Automatic assembly planning (AAP) has become an important element of manufacturing systems under the current status of CAD/CAM systems and the increasing emphasis on concurrent engineering (CE) and continuous acquisition and lifecycle support (CALS) (Hasin and Pandey, 1996). Commonly, AAP employs 2D engineering drawing and related assembly information to derive an APD diagram based on the contact-base or the feature-base relationships of the parts (Eng et al., 1999). De Fazio and Whitney adopted the concept of Bourjault (1984) to generate a complete set of assembly sequences (De Fazio and Whitney, 1987). They generated sequences in two stagescreating the precedence relations between liaisons or logical combinations of liaisons in a product and verifying liaison sequence. Kroll used graphbased procedures with conventional representations to reduce the number of sorting operations. $\mathrm{He}$ then extended his previous approach from uniaxial assemblies to triaxial assemblies and presented a set of rules for resolving conflicts between multiple parents and multiple offspring (Kroll, 1994). However, in practice most assembly companies use semi-automatic systems to generate an assembly plan and employ $2 \mathrm{D}$ cross-sectional views to represent their heuristic models (Lin and Chang, 1993a). An assembly plan is normally implemented with the planner's common sense, past experience, and limited knowledge. Although some mathematical algorithms have been proposed to generate possible sequences for a given assembly plan (Santochi and Dini, 1992), choosing an optimal assembly sequence in a complicated system is not feasible.

In practice, a common approach to assembly planning is "assembly by disassembling", i.e. an assembly sequence results from systematically disassembling the final product and reversing the disassembling sequence (Lee, 1989; Mosemann and Wahl, 2001). This recursive decomposition process may be represented by the non-ordered directed tree, which is called "assembly tree". It usually employs the contact-base feature or mating relations to represent the precedence relationships of the product. Mosemann and Wahl suggested that the assembly precedence could be formulated by using the skill primitives classified by the decomposition (Mosemann and Wahl, 2001). The limitation of many of these approaches is that they only consider partial assembly characteristics of a product to determine precedence constraints and sequences (Rajan and Nof, 1996). 
To enhance the manufacturability and quality of a product, all design goals and related constraints must be considered in the early design stage. The implementation of DFA and design for manufacture (DFM) led to enormous benefits, including the simplification of products, reduction of assembly product costs, improvement of quality, and reduction of time to market (Kuo et al., 2001). The research on the DFA was pioneered by Boothroyd and Dewhurst (1983). They adopted several basic criteria, a database of real-time standards, a DFA reference index, and identifications of assembly difficulties to evaluate the initial design and aimed at reducing the assembly costs. There are several successful examples in industry that exemplify the effectiveness of the DFA approach in the manufacturing environment (Wang and Trolio, 2001; Hsu et al., 1998; Dowlatshahi, 1995). Wang and Trolio used a clustered method to calculate the DFA index regarding the parts chosen for assembly (Wang and Trolio, 2001). Hsu et al. adopted a design concepts library to support the designers in synthesizing some feasible assembly alternatives in the design phase real-time (Hsu et al., 1998). Dowlatshahi focused on establishing an integrated manufacturing system design through querying the available methods from the database of standard operation procedures and standard times (Dowlatshahi, 1995). Indeed, these researches can improve the manufacturability potential of the assembly plan. However, they normally select a feasible design based on their domain knowledge or experience and lack of a systematic and integrated evaluating approach.

\section{Description of generation procedures}

The working concepts and procedures of the proposed approach are shown in Fig. 1. Initially, input detailed data from a $2 \mathrm{D}$ engineering drawing and related assembly information into a CAD assembly package. Secondly, the correct Explosion Graph was developed by using the transforming rules in Section 3.1. Finally, the relational models were generalized to represent the assembly precedence relations, and then an evaluating mechanism was employed to find a feasible solution. The planning process was recursive until the defined criteria is satisfied. The main outputs of the assembly planning were the complete relational model graph and the APD diagram.

\subsection{Deriving an Explosion Graph}

The Explosion Graph is the result of completely disassembling a product based on its geometric contact relations. This study used the "contactabove" concept, to construct the Above Graph, and then derived the Explosion Graph. "Above"

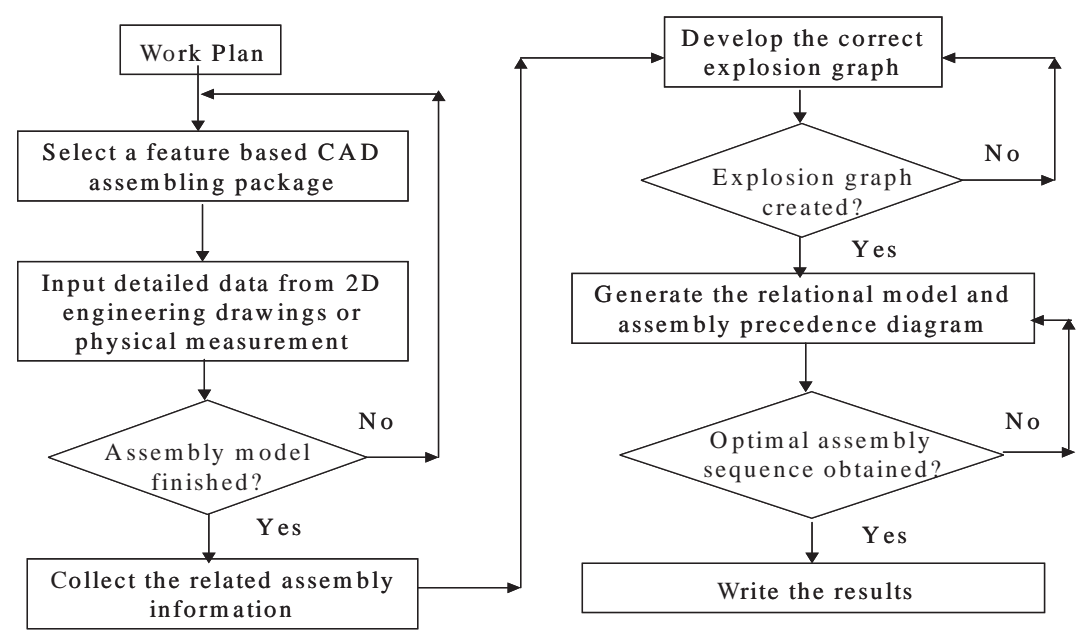

Fig. 1. Working concepts and procedures of assembly planning. 
implies that a part is located absolutely or relatively above the other parts in the $Z$-direction, or outside the other parts in the $X$ - $/ Y$-directions. Two assembly parts are generally related in one of the following ways: no contact (translatable), attached and fastened (non-translatable). If a component can be pre-assembled separately, this study took that component as a part and that need not be disassembled. Some transformation rules are used in this paper to define the "Above" relation of a component. The symbols ' $>$ ' and ' $\rightarrow$ ' are used to denote the 'above' and 'precede' relations in the deployment direction.

Rule 1: Set main deployment direction along the based part composed in that direction.

Rule 2: If $D \min (A)>D \min (B)$, where $D \min (A)$ and $D \min (B)$ are the min dimensions of parts $A$ and $\mathrm{B}$ along the deployment direction (see Fig. 2); then, the relation is defined as part $\mathrm{A}>$ part $\mathrm{B}$.

Rule 3: If $D \min (A)=D \min (B), \quad D \max (A)>$ $D \max (B), \quad$ where $\quad D \min (A), D \min (B), D \max (A)$ and $D \max (B)$ are the min and $\max$ dimensions of parts $\mathrm{A}$ and $\mathrm{B}$ along the deployment direction, then the relation is defined as part $\mathrm{A}>$ part $\mathrm{B}$.

Rule 4: If $D \min (A)=D \min (B), \quad D \max (A)=$ $D \max (B)$, and part $\mathrm{A}$ is larger than part B (in diameter, weight or volume), then the relation is defined as part $\mathrm{A}>$ part $\mathrm{B}$.

Rule 5: If part A is mounted on part $\mathrm{B}$ and intersects the deployment direction, then the relation is defined as part $\mathrm{A}>$ part $\mathrm{B}$.

Rule 6: If two components have the same priority according to Rules $2-4$, a component is selected according to a left-to-right and/or counter-clockwise sequence.

In practice, inference becomes more difficult as the product becomes more complex. This study

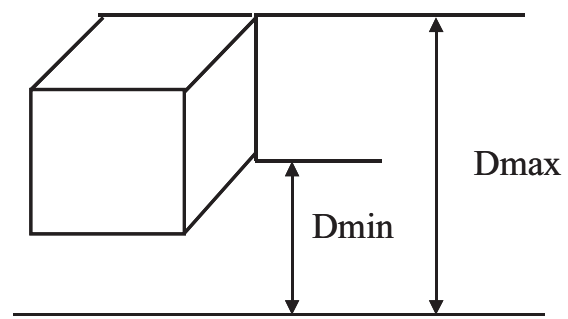

Fig. 2. Max- and min-dimensions of a part in deployment direction.

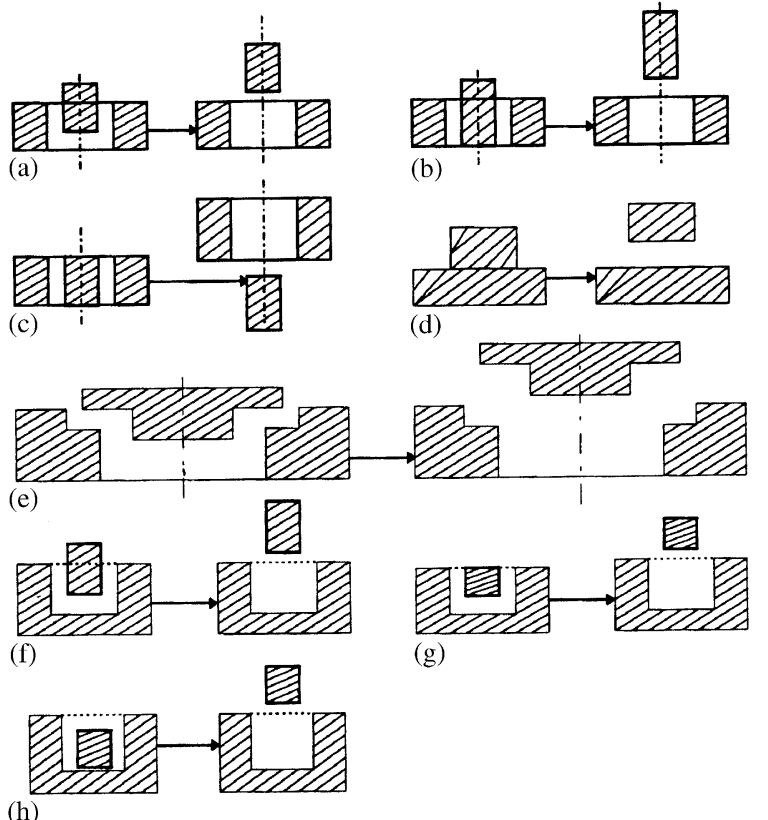

Fig. 3. Eight Above-Graph based sets.

used the contact-based Above Graph with the heuristic transformation rules mentioned above, to derive the Explosion Graph and simplify the inference process. Eight above-based sets, shown in Fig. 3, were used to guide the transformation. Although an APD tree can offer the effective route to assembly components and determines the best assembly sequence, generating feasible sequences for an assembly plan requires an experienced planner to address the following questions:

(1) How should a base part be selected?

(2) What are the precedence sequences among the contacted parts?

(3) What are the contact relationships (liaisons) between each of a pair of parts?

(4) Must the case be sub-assembled in advance?

\subsection{Creating a product data structure}

Assembly hierarchy is a conventional way of expressing a product data structure. Three levels of assembly hierarchy were used in this study - the top-relation model, the sub-relation model and the macro-relation model-to show the complete relationships of the product. 
To develop each level of the relational model, the base part must be identified and selected in advance. Correctly specifying a base part can reduce the number of generations in assembly planning. The following rules were used to identify and select the base part:

(1) The base part is initially selected to decompose a part's connectivity graph (PCG) into APD trees.

(2) The base part is the foundation of the APD trees.

(3) If there are more than two sub-assemblies, the non-moving one, the largest one, or the one that is combined with the largest number of parts, must be first selected as the base part.

(4) Any part which must be fixed by industrial fixture devices to support or stabilize the noncontacting parts, must be chosen as the base part.

\subsection{Development steps for the three-level relational model}

The relational model, like as the Explosion Graph, is also used to describe the assembly relations of the parts. It is a hierarchical structure for organizing the relations of the parts. The following steps were followed to develop threelevel relational model graphs and to create the APD trees:

(1) Select the entire top-level of sub-assemblies.

(2) Create the first level of the relational model. Draw out the next level of parts and subassemblies for each top-level of sub-assemblies according to the top-level of sub-assemblies.

(3) Create the second level of the relational model. Draw out the next level of parts and sub-assemblies for each upper level of subassemblies according to the upper level of subassemblies.

(4) Repeat Step 3 until the bottom level is reached.

(5) Create the macro-relational model graphs. Join each lower level relational model graph to the upper level relational model, to form macro-relational model graphs.
(6) Create the APD trees sequentially from the macro-relational model graphs according to the rules for selecting the base part and the transformation rules.

(7) Apply the RMST method to find the minimal precedence constraints of assembly.

\section{Mathematical model and the RMST algorithm}

Introducing graphical models to represent accurately the assembly diagrams is often relatively easy, because several efficient methods are available for handling them. The minimum spanning tree (MST) is a conventional way of deriving an optimal assembly sequence. Some well-known MST heuristic methods are often applied to derive the shortest path (Kruskal, 1956; Prim, 1957). In contrast to conventional methods such as linear programming and integer programming, which are NP-hard, a MST can be constructed in time, $O(n \log n)$ (Preparata and Shamos, 1988). This study adopted the RMST algorithm, proposed by Feng and $\mathrm{Yu}$ (1998), to derive minimal precedence constraints of assembly.

\subsection{Problem statement}

Consider a connected graph, $G=(V, E)$, where $V=\left\{v_{0}, v_{1}, \ldots, v_{n-1}\right\}$ is the set of vertices (components/parts), and $E \subseteq V \times V$ is the set of edges (assembly relations). MST (optimal assembly sequence) is a connected acyclic sub-graph of $G$ with minimum cost. The objective function can be expressed as follows:

Minimize TC $=\sum_{i=1}^{n} \sum_{j=1}^{n} P_{i j} \times X_{i j}$

subject to $X_{i j}= \begin{cases}1 & \text { if the sequence is selected, } \\ 0 & \text { otherwise. }\end{cases}$

where $P_{i j}$ is the assembly relation penalty between parts $i$ and $j$; $X_{i j}$ is $1(0)$ if the assembly sequence from parts $i$ to $j$ is selected (not selected), and TC is the total penalty in a given solution. The goal 
is to minimize the total penalty and meet the pre-defined precedent relations.

\subsection{Penalty matrix}

The penalty represents the cost incurred by assigning an unsuitable or unfeasible assembly sequence. Table 1 presents an illustrative example of the penalty weightings of some crucial factors. For instance, the penalty index is set 0 if part $i$ is absolutely located above part $j$; the penalty index is set 9 if the assembly relation is a "loose above" relation; the penalty index is set 999, if the assembly relation is prohibited. By employing the idea of penalty, other crucial factors concerning assembly planning, such as the frequency of changing tools, the similarity of assembly operations, the quality of assembly, the complexity of assembly, etc., can be considered simultaneously. Importantly, for avoiding the inaccurate estimation of the penalty of respective factors, it needs experienced engineers to perform the evaluation.

The penalty matrix of an assembly plan can be synthesized from the penalty index of respective crucial factors in Eq. (3). $p_{i j k}$ represents the penalty index of crucial factors between part $i$ and part $j$; $m$ is the number of crucial factors evaluated; and $w_{k}$ is the weighting of those crucial factors. In practice, the planner can decide to set the weightings to different values to meet business environment. Tables 2-4 present the penalty matrix of the study case. To meet the precedent relations of the assembly, a big penalty index can be used to avoid an inaccurate assignment

$P_{i j}=\sum_{k=1}^{m} w_{k} \times p_{i j k}$.

\subsection{Algorithm of RMST}

This study applied the RMST method to derive an optimal solution. The algorithm directly refers to the algorithm of Feng and $\mathrm{Yu}$ but adds some rules to prevent a cyclic solution. The RMST algorithm is summarized as follows:

Step 1: Identify each independent sub-assembly or part.

Step 2: Determine the penalty matrix for each sub-assembly or part.

Table 1

An example of penalty index

\begin{tabular}{lll}
\hline Penalty rank & Penalty index & Description \\
\hline 1 & 0 & Simple work, forced precedent sequence, direct or absolute above relation \\
2 & $1-5$ & A little difficulty, need careful operation, tools changing infrequently, close above relation \\
3 & $6-9$ & Very difficult, easily damage the component, tools changing frequently, loose above relation \\
4 & 999 & Prohibit, not any relation \\
\hline
\end{tabular}

Table 2

Penalty matrix of SACS and assigning sequences

\begin{tabular}{lrrrrrrrr}
\hline & PC1 & DE2 & PH3 & BC4 & CC5 & SD6 & RR7 & Minimum \\
\hline PC1 & 999 & 2 & 6 & 1 & 0 & 9 & 9 & 9 \\
DE2 & 20 & 999 & 2 & 9 & 9 & 9 & 9 & 0 \\
PH3 & 6 & 3 & 999 & 6 & 9 & 9 & 9 & 0 \\
BC4 & 2 & 9 & 9 & 999 & 4 & 999 & 999 & 0 \\
CC5 & 999 & 999 & 999 & 999 & 999 & 6 & 4 & 0 \\
SD6 & 999 & 999 & 999 & 999 & 0 & 999 & 5 \\
RR7 & 999 & 999 & 999 & 999 & 6 & 0 & 999 & 0 \\
\hline
\end{tabular}


Table 3

Penalty matrix of SAPE and assigning sequences

\begin{tabular}{lrrrrrrr}
\hline & FS8 & TR9 & TF10 & SH11 & PE12 & PT13 & Minimum \\
\hline FS8 & 999 & 10 & 6 & 6 & 6 & 6 & 0 \\
TR9 & 0 & 999 & 20 & 5 & 6 & 6 & 6 \\
TF10 & 6 & 1 & 999 & 999 & 0 & 6 & 0 \\
SH11 & 9 & 9 & 0 & 0 & 999 & 4 & 2 \\
PE12 & 9 & 9 & 6 & 6 & 0 & 999 & 0 \\
PT13 & 9 & 9 & 6 & & & 0 \\
\hline
\end{tabular}

Table 4

The penalty matrix of SAHD and assigning sequences

\begin{tabular}{|c|c|c|c|c|c|c|c|c|c|}
\hline & R3_14 & SCR15 & SCP16 & R2_17 & SA18 & SA19 & SA20 & $\mathrm{R} 1 \_21$ & Minimum row \\
\hline R3_14 & 999 & ${ }^{1} 0$ & 6 & 9 & 9 & 9 & 9 & 9 & 0 \\
\hline SCR 1 & 0 & 999 & ${ }^{2} 0$ & 6 & 9 & 9 & 9 & 9 & 0 \\
\hline SCP1 & 6 & 0 & 999 & ${ }^{3} 0$ & 9 & 9 & 9 & 9 & 0 \\
\hline R2_17 & 6 & 6 & 0 & 999 & 3 & 3 & $\begin{array}{ll}6 & 3\end{array}$ & 6 & 0 \\
\hline SA18 & 9 & 9 & 3 & 6 & 999 & 3 & 3 & $\begin{array}{l}74 \\
{ }^{7}\end{array}$ & 3 \\
\hline SA19 & 9 & 9 & 3 & 6 & $\begin{array}{ll}4 & 0\end{array}$ & 999 & 3 & 4 & 0 \\
\hline SA20 & 9 & 9 & 3 & 6 & 0 & 52 & 999 & 4 & 0 \\
\hline R1_21 & 9 & 9 & 9 & 5 & 3 & 5 & 3 & 999 & 3 \\
\hline
\end{tabular}

Step 3: Select the minimum penalty from each row and mark the smallest one from the chosen penalty and assign it to $P_{i j}$. For example, if two cells have the same minimum value, select one using the top-left to bottom-right criterion.

Step 4: Assign the assembly sequence from part $i$ to part $j$ and mark a line on the $i$ th row and the $j$ th column of the matrix; simultaneously change the $P_{j i}$ penalty (part $j$ to part $i$ ) to 999 (for preventing cyclic assignment).

Step 5: Repeat Steps 3 and 4 until the number of marking lines equals $n-1$ (where $n$ is the total number of assembly parts) or until all parts are selected.

Step 6: Detect all assigned relations.

6-1. If no cycle tree exists, go to Step 7.

6-2. If a cycle tree exists, then de-cycle it into a single tree with minimum penalty, then return to Step 3. (Note: The cycle normally comes from the subassembly. If it is a case of subassembly and this component can be assembled separately, the Step 6-2 can be omitted.)

Step 7: Re-connect all sub-trees into a single tree.

\section{A case study}

In this section, the example of a $120 \mathrm{~mm}$ APDSDS-T KE cartridge was used to demonstrate the generating procedures of assembly planning. Fig. 4 shows the part lists and assembly codes of the case study.

\subsection{Validating the accuracy of the exploded view}

Applying a correct exploded view allows us to derive the exact assembly plans. The validity of each exploded view can be confirmed by the contact relationships of the spatial structure and the transformation rules.

\subsection{Creating the Above Graph}

The Above Graph can be directly created from the exploded view, which possesses the contact relationships of a spatial structure. Fig. 5 shows the exploded views of the study case. In this case three Sabot-parts (SA18, SA19 and SA20) can be combined into a sub-component of SABOT along 
$X-Y$ direction. Fig. 6 shows the created outcome of the Above Graph (while considering only the $Z$ direction and reserving SABOT as a sub-assembly).

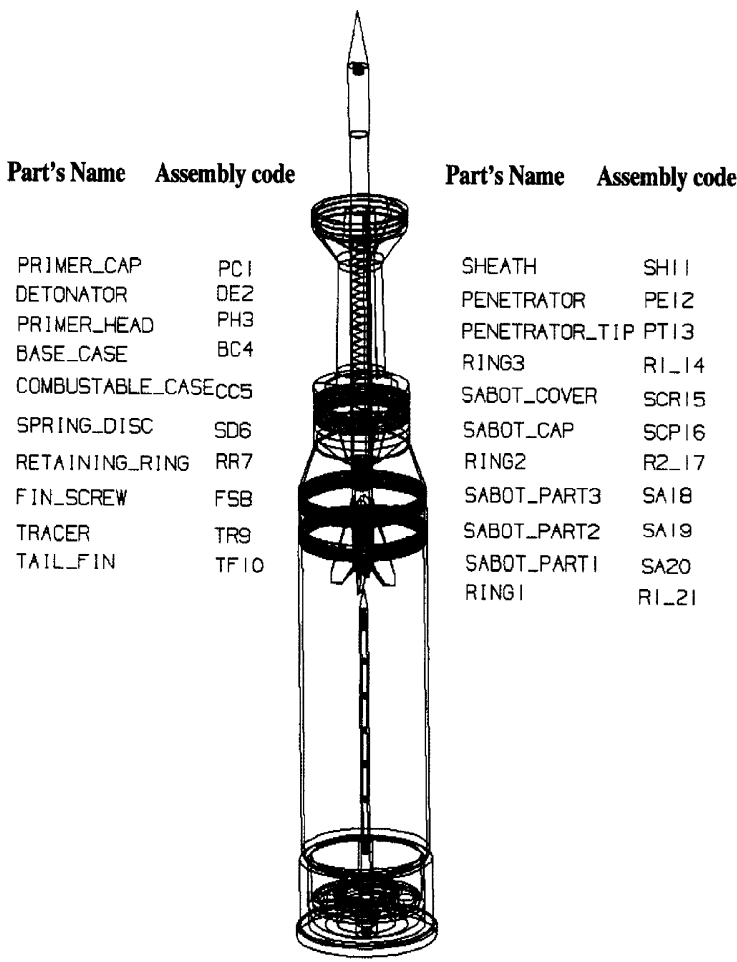

Fig. 4. Part names and assembly codes of $120 \mathrm{~mm}$ APDSDS-T KE cartridge.

\subsection{Explosion Graph transformation}

The Above Graph can be transformed into the Explosion Graph by the transformation rules depicted in Section 3. The transforming procedures convert the branched type of Above Graph into the one of linear type. The transforming procedures are summarized as follows:

1. First transformation: According to the relationships of PH3 $\rightarrow$ BC4, RR7 $\rightarrow$ P3_14, and PT13 $\rightarrow$ SABOT, Fig. 6 can be converted into Fig. 7a.

2. Second transformation: Owing to the branched form existing in Fig. 7a, the Above relationship

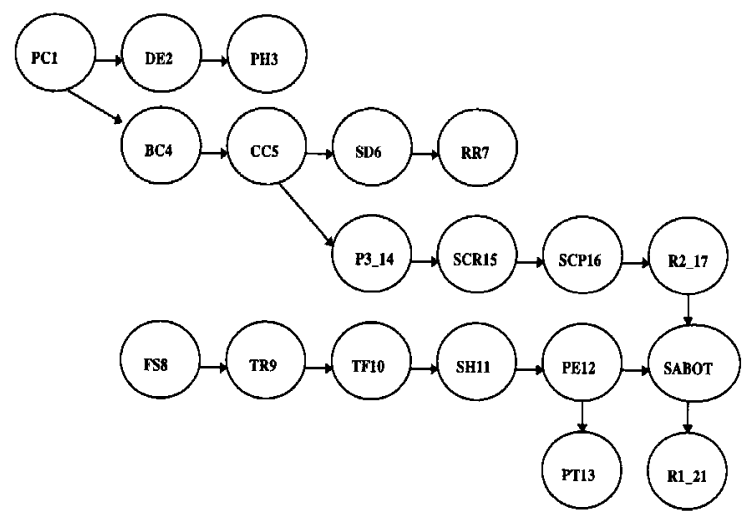

Fig. 6. The Above Graph of the study case.

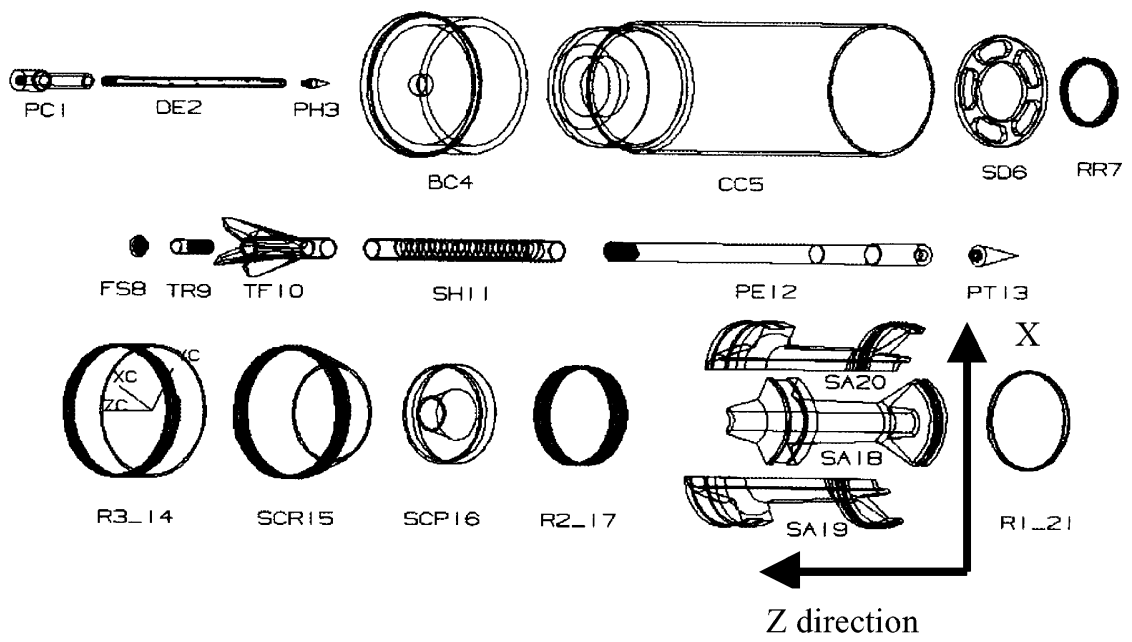

Fig. 5. The exploded view of the study case. 

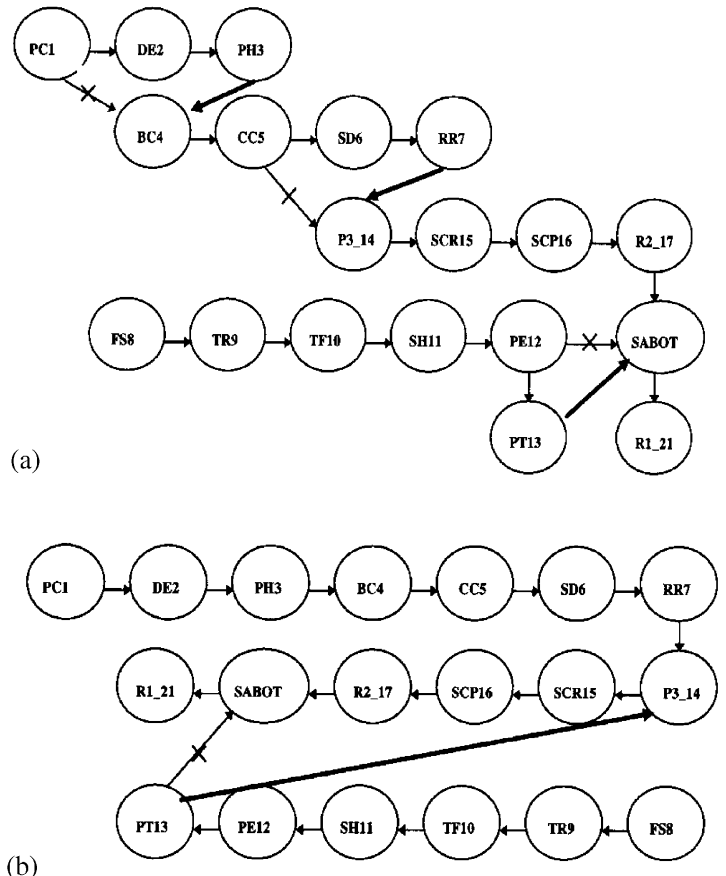

(b)

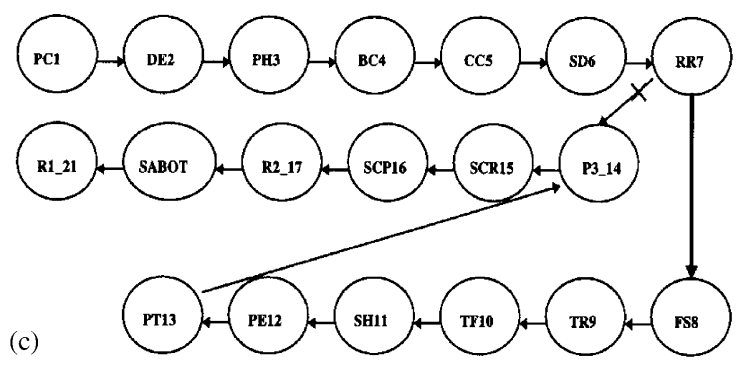

Fig. 7. Three transforming steps of the Explosion Graph.

in PT13 $\rightarrow$ R3_14 must be converted. Fig. 7b shows the converted outcome.

3. Third transformation: In Fig. 7b, the branched form that must be converted again still exists. According to the Above relationship of RR7 $\rightarrow$ FS8, it can be converted as shown in Fig. 7c to obtain a complete Above Graph.

\subsection{Developing relational model graphs and APD trees}

Three symbols are used to express the relationships of the combined parts. The square denotes
Base component: SUBASSY_HEAD(SAHD)

Where

SACS: SUBASSY_CASE

SAPE: SUBASSY_PENETRATOR
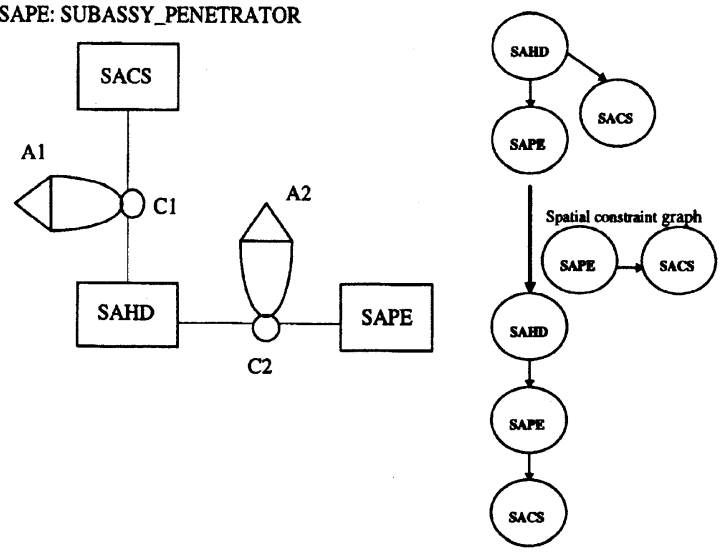

Fig. 8. The top-relational model graphs and APD trees.

the part name to be combined. The circle represents the contact relation between the two combined parts. Meanwhile, the triangle refers to the attachment relationship.

Fig. 8 shows the top-relational model graph of the study case. The graph is directly derived from the top of sub-assemblies shown in Fig. 5. The three sub-assemblies shown in Fig. 8, SAHD, SACS and SAPE, should be extended to the second level of relational model. For brevity, the other two relational models of the sub-assembly, SACS and SAPE, are omitted.

While completing the second and lower level relational model graphs of each sub-assembly, the macro-relational model graphs can be constructed by joining each of the lower sub-relational model graphs from bottom to top. Fig. 9 shows the complete macro-relational model graphs and APD trees. By mapping the contact relationships in Fig. 9, the assembly hierarchal relationships can be expressed as an incidence matrix as shown in Fig. 10.

\subsection{Minimization of precedence constraints}

The study case be decomposed into three independent sub-assemblies: SACS, SAPE and 


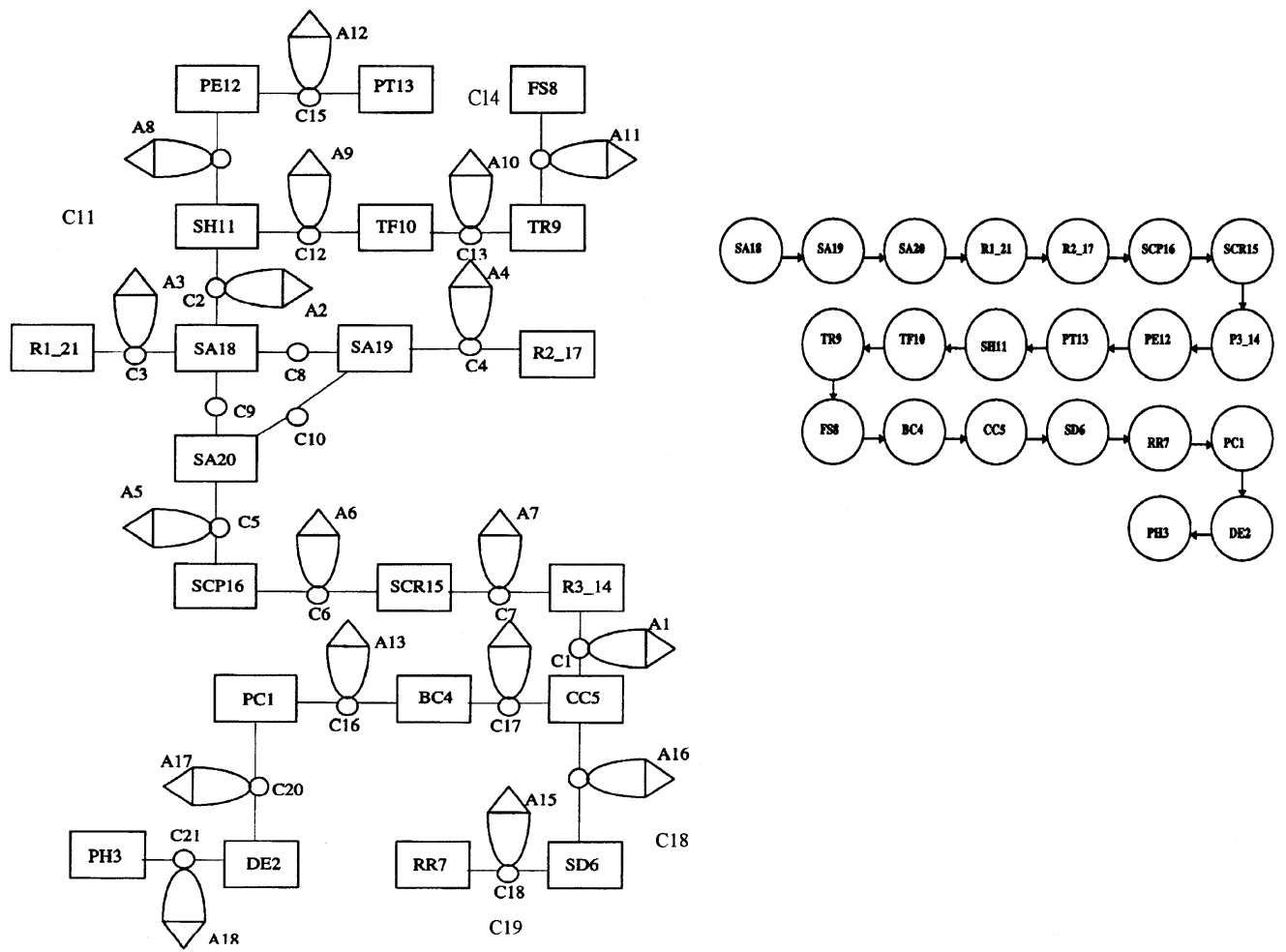

Fig. 9. The complete relational model graphs and the APD trees of the study case.

SAHD. The three tables detailed in Tables $2-4$ are used to express the penalty matrix and obtain the minimal precedence constraints for each subassembly. All sub-trees are then re-connected to form final results.

(1) The results of assigning SACS (see Table 2):

$\mathrm{PC} 1 \rightarrow \mathrm{BC} 4, \mathrm{DE} 2 \rightarrow \mathrm{PC} 1, \mathrm{PH} 3 \rightarrow \mathrm{DE} 2$,

$\mathrm{BC} 4 \rightarrow \mathrm{CC} 5, \mathrm{SD} 6 \rightarrow \mathrm{RR} 7, \mathrm{CC} 5 \rightarrow \mathrm{SD} 6$

The results of re-connecting all sub-trees:

$$
\begin{aligned}
& \mathrm{PH} 3 \rightarrow \mathrm{DE} 2 \rightarrow \mathrm{PC} 1 \rightarrow \mathrm{BC} 4 \rightarrow \mathrm{CC} 5 \rightarrow \\
& \mathrm{SD} 6 \rightarrow \mathrm{RR} 7
\end{aligned}
$$

(2) The results of assigning SAPE (see Table 3):

FS8 $\rightarrow$ TR9, TR9 $\rightarrow$ TF10, SH11 $\rightarrow$ PE12,

$\mathrm{PE} 12 \rightarrow \mathrm{PT} 13, \mathrm{TF} 10 \rightarrow \mathrm{SH} 11$

The results of re-connecting all sub-trees:

$\mathrm{FS} 8 \rightarrow \mathrm{TR} 9 \rightarrow \mathrm{TF} 10 \rightarrow \mathrm{SH} 11 \rightarrow \mathrm{PE} 12 \rightarrow \mathrm{PT} 13$
(3) The results of assigning SAHD (see Table 4):

R3_14 $\rightarrow$ SCR15, SCR15 $\rightarrow$ SCP16, $\mathrm{SCP} 16 \rightarrow \mathrm{R} 2$ 17, SA19 $\rightarrow \mathrm{SA} 18, \mathrm{SA} 20 \rightarrow \mathrm{SA} 19$, $\mathrm{R} 2 \_17 \rightarrow \mathrm{SA} 20, \mathrm{SA} 18 \rightarrow \mathrm{SR} 1 \_21$

The results of re-connecting all sub-trees:

$$
\begin{aligned}
& \mathrm{R} 3 \_14 \rightarrow \mathrm{SCR} 15 \rightarrow \mathrm{SCP} 16 \rightarrow \mathrm{R} 2 \_17 \rightarrow \\
& \mathrm{SA} 20 \rightarrow \mathrm{SA} 19 \rightarrow \mathrm{SA} 18 \rightarrow \mathrm{SR} 1 \_21
\end{aligned}
$$

(4) The final results of re-connecting all subassemblies:

$$
\begin{aligned}
& \mathrm{PH} 3 \rightarrow \mathrm{DE} 2 \rightarrow \mathrm{PC} 1 \rightarrow \mathrm{BC} 4 \rightarrow \mathrm{CC} 5 \rightarrow \mathrm{SD} 6 \rightarrow \\
& \mathrm{RR} 7 \rightarrow \mathrm{FS} 8 \rightarrow \mathrm{TR} 9 \rightarrow \mathrm{TF} 10 \rightarrow \mathrm{SH} 11 \rightarrow \\
& \mathrm{PE} 12 \rightarrow \mathrm{PT} 13 \rightarrow \mathrm{R} 3 \_14 \rightarrow \mathrm{SCR} 15 \rightarrow \mathrm{SCP} 16 \rightarrow \\
& \mathrm{R} 2 \_17 \rightarrow \mathrm{SA} 20 \rightarrow \mathrm{SA} 19 \rightarrow \mathrm{SA} 18 \rightarrow \mathrm{R} 1 \_21
\end{aligned}
$$

Total penalty is fifteen. 


\begin{tabular}{|l|l|l|l|l|l|l|l|l|l|l|l|l|l|l|l|l|l|l|l|l|l|l|}
\hline & PC1 & DE2 & PH3 & BC4 & CC5 & SD6 & RR7 & FS8 & TR9 & $\begin{array}{l}\text { FF } \\
10\end{array}$ & $\begin{array}{l}\text { SH } \\
11\end{array}$ & $\begin{array}{l}\text { PE } \\
12\end{array}$ & $\begin{array}{l}\text { PT } \\
13\end{array}$ & $\begin{array}{l}\text { R3 } 3 \\
14\end{array}$ & $\begin{array}{l}\text { SCR } \\
15\end{array}$ & $\begin{array}{l}\text { SCP } \\
16\end{array}$ & $\begin{array}{l}\text { R2 } \\
17\end{array}$ & $\begin{array}{l}\text { SA } \\
18\end{array}$ & $\begin{array}{l}\text { SA } \\
19\end{array}$ & $\begin{array}{l}\text { SA } \\
20\end{array}$ & $\begin{array}{l}\text { R1 } \\
21\end{array}$ \\
\hline PC1 & & C20 & & C16 & & & & & & & & & & & & & & & & & \\
\hline DE2 & C20 & & C21 & & & & & & & & & & & & & & & & & & \\
\hline PH3 & & C21 & & & & & & & & & & & & & & & & & & & \\
\hline BC4 & C16 & & & & C17 & & & & & & & & & & & & & & & & \\
\hline CC5 & & & & C17 & & C18 & & & & & & & & C1 & & & & & & & \\
\hline SD6 & & & & & C18 & & C19 & & & & & & & & & & & & & & \\
\hline RR7 & & & & & & C19 & & & & & & & & & & & & & & & \\
\hline FS8 & & & & & & & & & C14 & & & & & & & & & & & & \\
\hline TR9 & & & & & & & & C14 & & C13 & & & & & & & & & & & \\
\hline TF10 & & & & & & & & & C13 & & C12 & & & & & & & & & & \\
\hline SH11 & & & & & & & & & & C12 & & C11 & & & & & & C2 & & & \\
\hline PE12 & & & & & & & & & & & C11 & & C15 & & & & & & & & \\
\hline PT13 & & & & & & & & & & & & C15 & & & & & & & & & \\
\hline R3_14 & & & & & C1 & & & & & & & & & & C7 & & & & & & \\
\hline SCR15 & & & & & & & & & & & & & & C7 & & C6 & & & & & \\
\hline SCP16 & & & & & & & & & & & & & & & C6 & & & C5 & C5 & C5 & \\
\hline R2_17 & & & & & & & & & & & & & & & & & & C4 & C4 & C4 & \\
\hline SA18 & & & & & & & & & & & C2 & & & & & C5 & C4 & & C2 & C2 & C3 \\
\hline SA19 & & & & & & & & & & & & & & & C5 & C4 & C2 & & C10 & C3 \\
\hline SA20 & & & & & & & & & & & & & & & C5 & C4 & C2 & C10 & & C3 \\
\hline R1_21 & & & & & & & & & & & & & & & & & & C3 & C3 & C3 & \\
\hline
\end{tabular}

Fig. 10. The incidence matrix of assembly relations.

\section{Discussion and conclusions}

This paper offers a three-stage integrated approach and proposes a RMST algorithm to assist planners to find an optimal assembly plan. Theoretically, an assembly plan can be optimized based on the factor of assembly time. However, it is an uncertainty factor before the determination of the assembly method and the completion of the jig and fixture. This paper proposes a penalty index to express the complexity of the assembly relations, the cost or the time consumed by these operations (tool, machine, etc.) in the design phase, to evaluate the feasibility of respective assembly sequences. The penalty is the excess cost incurred if an unsuitable or unfeasible assembly sequence is assigned. The penalty matrix of an assembly plan can be synthesized from the penalty index of crucial relevant assembly factors. Using the penalty rank method allows the designer to simultaneously evaluate the crucial factors for meeting the multi-criteria of assembly planning problem.
In practice, the contact-base feature is usually employed to represent the precedence relationships of the product. However, the contact-base precedence diagram cannot effectively express the complexity of the assigned assembly relations. The results generated for the case study verify the feasibility of the proposed approach. The proposed approach facilitates the DFA in potential applications of 3D component models to assist manual or automatic assembly in a virtual environment, and allow the designer to recognize the relative position, constraints and relationships of the 3D components using graph-oriented methods: Above Graph, APD and relational model graph. A planner can generate a correct Explosion Graph and construct an incidence matrix for validating the assembly relations through applying Above Graph and relation models. This method guarantees the quality of the generated assembly plan. The planner can reexamine the fitness for assembly of a given product, by evaluating the penalties associated with the assembly relations enhancing the 
product's fitness for assembly, during the design phase.

\section{References}

Amen, M., 2000. Heuristic methods for cost-oriented assembly line balancing: A survey. International Journal of Production Economics 68 (1), 1-14.

Amen, M., 2001. Heuristic methods for cost-oriented assembly line balancing: A comparison on solution quality and computing time. International Journal of Production Economics 69 (3), 255-264.

Boothroyd, G., Dewhurst, P., 1983. Design for assembly-a designers handbook. Technique Report, Department of Mechanical Engineering, University of Massachusetts.

Bourjault, A., 1984. Contribution à une approche méthodologique de l'assemblage automatisé: Elaboration automatique des séquences opératoires, Ph.D. Thesis, Faculté des Sciences et des Techniques de l'Université de Franche-Comté, France.

Brian, T.F., Patterson, J.H., 1984. An integer programming algorithm with network cuts for solving the assembly line balancing problem. Management Science 30 (1), 85-99.

De Fazio, T.L., Whitney, D.E., 1987. Simplified generation of all mechanical assembly sequence. IEEE Journal of Robotics and Automation RA 3 (6), 640-658.

Dowlatshahi, S., 1995. An integrated manufacturing system design: An applied approach. International Journal of Production Economics 42, 187-199.

Elsayed, E.A., Boucher, T.O., 1994. Analysis and Control of Production Systems. Prentice-Hall International Series in Industrial and Systems Engineering, New Jersey.

Eng, T.H., Ling, Z.K., Olson, W., McLean, W.C., 1999. Feature-based assembly modeling and sequence generation. Computers \& Industrial Engineering 36, 17-33.

Feng, J.W., Yu, P.L., 1998. Minimum spanning table and optimal expansion of competence set. Journal of Optimization Theory and Applications 99 (3), 655-679.

Hackman, S.T., Magazine, M.J., Wee, T.S., 1989. Fast, effective algorithms for assembly line balancing problems. Journal of Operational Research 37 (6), 916-924.

Hasin, M.A., Pandey, P.C., 1996. Analysis of present scheme of MRP II-CAD integration: Problems and prospects. International of Computer Applications in Technology 9 (2-3), 126-130.

Helgeson, W.P., Birnie, D.P., 1961. Assembly line balancing using the ranked positional weight technique. Journal of Industrial Engineering 12 (6), 394-398.

Hsu, W., Fuh, J.Y.H., Zhang, Y., 1998. Synthesis of design concepts from a design for assembly perspective. Computer Integrated Manufacturing Systems 11 (1-2), 1-13.

Kilbridge, M.D., Wester, L., 1961. A heuristic method of assembly line balancing. Journal of Industrial Engineering 12 (4), 292-298.
Kim, Y.K., Kim, Y.J., Kim, Y.H., 1996. Genetic algorithm for assembly line balancing with various objectives. Computers and Industrial Engineering 30 (3), 397-409.

Kroll, E., 1994. Intelligent assembly planning on triaxial products. Concurrent Engineering: Research and Applications 1 (2), 311-319.

Kruskal, J.B., 1956. On the shortest spanning tree of a graph and the traveling salesman problem. Proceedings of American Mathematical Society 7, 48-50.

Kuo, T.C., Huang, S.H., Zhang, H.C., 2001. Design for manufacture and design for ' $\mathrm{X}$ ': Concepts, applications, and perspectives. Computers \& Industrial Engineering 41, 241-260.

Lazzerini, B., Marcelloni, F., 2000. A genetic algorithm for generating optimal assembly plans. Artificial Intelligence in Engineering 14, 319-329.

Lee, S., 1989. Disassembly planning by subassembly extraction. Proceedings of the Third ORSA/TIMS Conference on Flexible Manufacturing Systems. Cambridge, MA, pp. 383-388.

Leu, Y.Y., Matheson, L.A., Rees, L.P., 1996. Sequencing mixed-model assembly lines with genetic algorithms. Computers and Industrial Engineering 30 (4), 1027-1036.

Lin, A.C., Chang, T.C., 1993a. An integrated approach to automated assembly planning for three-dimensional mechanical products. International Journal of Production Research 31 (5), 1201-1227.

Lucertini, M., Pacciarelli, D., Pacifici, A., 1998. Modeling an assembly line for configuration and flow management. Computer Integrated Manufacturing Systems 11 (1-2), $15-24$.

Mosemann, H., Wahl, F., 2001. Automatic decomposition of planned assembly sequences into skill primitives. IEEE Transaction on Robotics and Automation 17 (5), 709-718.

Nof, S.Y., Wilhelm, W.E., Warnecke, H.J., 1997. Industrial Assembly. Chapman \& Hall, London.

Ponnambalam, S.G., Aravindan, P., Naidu, G.M., 2000. A multi-objective genetic algorithm for solving assembly line balancing problem. Advanced Manufacturing Technology $16,341-352$.

Preparata, F.P., Shamos, M.I., 1988. Computational Geometry: An Introduction, 2nd Edition. Springer, New York.

Prim, R.C., 1957. Shortest connection networks and some generations. Bell System Technical Journal 36, 1389-1401.

Rajan, V.N., Nof, S.Y., 1996. Minimumal precedence constraints for integrated assembly and execution planning. IEEE Transactions on Robotics and Automation 12 (2), 175-186.

Ramos, C., Rocha, J., Vale, Z., 1998. On the complexity of precedence graphs for assembly and task planning. Computers in Industry 36, 101-111.

Santochi, M., Dini, G., 1992. Computer-aided planning of assembly operations: The selection of assembly sequences. Robotics and Computer Intergrated Manufacturing 9 (6), 439-446. 
Song, D.P., Earl, C.F., Hicks, C., 2001. Stage due date planning for multistage assembly systems. International Journal of Production Research 39 (9), 1943-1954.

Tai, P.H., 1997. Feature-based assembly modeling for assembly sequence planning of three-dimensional products. M.Sc. Thesis. Cranfield University, UK.
Wang, J.H., Trolio, M., 2001. Using clustered assembly elements in the estimation of potential design for assembly benefits. International Journal of Production Research 39 (9), 1885-1895. 